\title{
Acute changes in short-term plasticity at synapses with elevated levels of neuronal calcium sensor-1
}

\author{
Tanya Sippy ${ }^{1,4}$, Alberto Cruz-Martín ${ }^{1,2}$, Andreas Jeromin ${ }^{3}$, and Felix E Schweizer ${ }^{1,2,5}$ \\ ${ }^{1}$ Department of Neurobiology and The Brain Research Institute, David Geffen School of Medicine \\ at UCLA, 650 Charles E. Young Drive South, Los Angeles, California 90095, USA \\ 2Interdepartmental Ph.D. Program for Neuroscience, UCLA, Los Angeles, California 90095, USA \\ ${ }^{3}$ Division of Neuroscience, Baylor College of Medicine, Houston, Texas 77030, USA
}

\section{Abstract}

Short-term synaptic plasticity is a defining feature of neuronal activity, but the underlying molecular mechanisms are poorly understood. Depression of synaptic activity might be due to limited vesicle availability, whereas facilitation is thought to result from elevated calcium levels. However, it is unclear whether the strength and direction (facilitation versus depression) of plasticity at a given synapse result from preexisting synaptic strength or whether they are regulated by separate mechanisms. Here we show, in rat hippocampal cell cultures, that increases in the calcium binding protein neuronal calcium sensor-1 (NCS-1) can switch paired-pulse depression to facilitation without altering basal synaptic transmission or initial neurotransmitter release probability. Facilitation persisted during high-frequency trains of stimulation, indicating that NCS-1 can recruit 'dormant' vesicles. Our results suggest that NCS-1 acts as a calcium sensor for short-term plasticity by facilitating neurotransmitter output independent of initial release. We conclude that separate mechanisms are responsible for determining basal synaptic strength and short-term plasticity.

Synaptic strength is dynamic and changes on the basis of specific input patterns and past history, in a process known as synaptic plasticity. Long-term plasticity lasting hours to days is thought to underlie processes of learning and memory, whereas short-term plasticity in the range of milliseconds to minutes is important for neurons to perform computational tasks, produce appropriate output in response to acute changes in activity, and initiate the changes necessary for long-term plasticity ${ }^{1-3}$. Although the rules governing short-term plasticity are not understood, a general trend is emerging: the direction of short-term plasticity (depression or facilitation) correlates with the initial efficacy with which synapses transduce action potentials into neurotransmitter release ${ }^{4}$. Synapses with a high release probability are more likely to show depression, consistent with a depletion of vesicles from the readily releasable pool $^{5,6}$ or an activity-dependent inhibition of the release machinery ${ }^{7}$. Synapses with an initially low release probability do not exhaust their releasable pool of vesicles in response to the first action potential, so a lingering effect of the first stimulation (most likely residual calcium) can potentiate the response to subsequent stimuli ${ }^{8}$. It thus remains unclear whether

\section{(C) 2003 Nature Publishing Group}

${ }^{5}$ Correspondence should be addressed to F.E.S. (felixs@ucla.edu).

${ }^{4}$ Present Address: MD-PhD Program, College of Physicians and Surgeons, Columbia University, 630 West 168 Street, New York, New York 10032, USA.

\section{COMPETING INTERESTS STATEMENT}

The authors declare that they have no competing financial interests. 
short-term plasticity simply reflects basal synaptic strength or whether it can be regulated by an independent mechanism.

Several models have been proposed to account for short-term facilitation, including summation of residual calcium with repetitive stimulation ${ }^{9,10}$ and local saturation of calcium buffers ${ }^{11,12}$. It has also been suggested that an additional calcium binding site(s) acts cooperatively with the main release sensor, although this site has not yet been identified ${ }^{13}$. Presynaptic calcium thus has a critical role as mediator of short-term plasticity, but its precise function and the identity of its downstream target(s) remain elusive $e^{4,8,14}$. Manipulations that affect short-term plasticity frequently alter calcium signaling and thereby affect basal transmitter release as well. It has therefore been difficult to determine whether short-term plasticity and basal transmitter release depend on common or distinct calcium signaling pathways. NCS-1, the mammalian homolog of the Drosphila protein frequenin ${ }^{15}$, has key features for linking residual calcium in the presynaptic terminal to changes in shortterm plasticity. It is a $22-\mathrm{kDa}$ cytoplasmic, neuron-specific protein that binds calcium in the submicromolar range via EF-hands ${ }^{16}$. Overexpression of the protein at the Drosophila melanogaster ${ }^{15}$ or Xenopus laevis ${ }^{17}$ neuromuscular junction caused a large facilitation of synaptic transmission. In both PC12 cells and chromaffin cells, NCS-1 enhances release in response to ATP, but not in response to direct depolarization. NCS-1 has been shown to regulate P/Q-type calcium currents at the calyx of Held ${ }^{18}$, but thus far, it has remained untested whether NCS-1 has a direct, specific effect on neurotransmitter release in the mammalian CNS.

We tested the effects of NCS-1 on synaptic transmission and short-term plasticity in hippocampal neurons. Consistent with previous reports, cultured hippocampal neurons showed paired-pulse depression, in contrast to the paired-pulse facilitation observed in vivo and in acute slices. We now report that transfection of NCS-1 into cultured hippocampal neurons switches the direction of short-term plasticity from depression to facilitation. This switch occurs without a change in basal release characteristics and without alterations of calcium currents ${ }^{18}$. Lowering extracellular calcium alleviates the depression in control neurons and increases the facilitation in NCS-1 transfected neurons, suggesting that NCS-1 specifically enhances facilitation rather than decreasing depression. Our results identify NCS- 1 as a calcium sensor for short-term plasticity in the mammalian nervous system and demonstrate that independent mechanisms exist for setting basal release levels and shortterm plasticity characteristics.

\section{RESULTS}

\section{NCS-1 changes paired-pulse plasticity}

We transfected dissociated hippocampal neurons cultured at low density with a plasmid encoding both NCS-1 and GFP (pNCS1-IRES-GFP; see Methods). Pairs of synaptically connected neurons were voltage-clamped at $-70 \mathrm{mV}$, an escape action potential was elicited in one of them by depolarizing it briefly $(<1 \mathrm{~ms})$ to +20 or $+50 \mathrm{mV}$, and the resulting excitatory postsynaptic current (EPSC) was measured in the other neuron (Fig. 1a,b). Many transfected/non-transfected neuron pairs had reciprocal connections, such that excitatory postsynaptic currents (EPSCs) could be recorded from both cells during the same experiment, giving an extremely useful internal control for potential homeostatic changes (Fig. 1b). In addition, we were able to distinguish pre- and postsynaptic effects of transfection in the same cell pair. Here we will refer to those recordings in which the stimulated (i.e., presynaptic) neuron did not express the transfected construct as 'control synapses' and those in which the stimulated neuron was transfected as 'NCS-1 synapses'. NCS-1 synapses were identified by a green fluorescent protein (GFP)-positive phenotype. In agreement with previous reports ${ }^{19-21}$, we observed robust paired-pulse depression (PPD) at 
control synapses (Fig. 1b,c). NCS-1 transfected synapses, however, showed strong and consistent paired-pulse facilitation (PPF) (Fig. 1b,d). This switch from depression to facilitation was evident over the entire time course of the recording, and although considerable trial-to-trial variability was evident, depression was reliably observed for control synapses and facilitation for NCS-1 synapses (Fig. 1c,d). Occasional autaptic connections (Fig. 1b, asterisks) showed plasticity congruent with their origin (presynaptic phenotype), providing yet another internal control for the specificity of the NCS-1 effect.

The vesicle-depletion model of PPD predicts that depression from trial to trial should correlate with the magnitude of the first response. However, when plotting the amplitude of the second response against the amplitude of the first response, we found no correlation between the two amplitudes for control synapses (Fig. 1e, circles; slope, $-0.01 \pm 0.05$; range, -0.196 to $0.238 ; r^{2}=0.02 \pm 0.02$; range, 0 to $0.06, n=9$ ) or NCS- 1 transfected synapses (Fig. 1e, triangles; slope $=0.193 \pm 0.05$; range, -0.008 to $0.377 ; r^{2}=0.07 \pm 0.03$; range, 0 to $0.28, n=8)^{7}$. This suggests that depression is not simply due to vesicle depletion. In addition, the average paired pulse ratio (PPR), measured as the ratio EPSC2/EPSC1, did not correlate with the initial amplitude, but it did strongly correlate with NCS-1 expression: depression for control synapses (PPR $<1$ ) and facilitation for NCS-1 transfected synapses (PPR > 1; Fig. 1f). This indicates that the facilitation conferred by NCS-1 expression is seen in both strongly and weakly coupled cell pairs.

We next studied the time course of short-term plasticity by varying the interstimulus interval (ISI). In control cells, depression was strongest at short intervals, showed partial recovery at $200 \mathrm{~ms}$ ISI and near-full recovery at $500 \mathrm{~ms}$ (Fig. 2b, open circles; $\mathrm{PPR}_{50 \mathrm{~ms}}, 0.56 \pm 0.09$; $\left.\mathrm{PPR}_{100 \mathrm{~ms}}, 0.59 \pm 0.04 ; \mathrm{PPR}_{200 \mathrm{~ms}}, 0.79 \pm 0.06 ; \mathrm{PPR}_{500 \mathrm{~ms}}, 0.91 \pm 0.09\right)$. A control plasmid coding only for GFP and lacking NCS-1 did not alter synaptic transmission (PPR $50 \mathrm{~ms}, 0.64$ $\pm 0.09, n=6, P>0.1$, data not shown). At NCS-1 transfected synapses, on the other hand, we observed PPF at ISIs up to $200 \mathrm{~ms}$ (Fig. 2a,b). Facilitation was greatest at the shortest interval (Fig. 2b, filled triangles; $\mathrm{PPR}_{20 \mathrm{~ms}}, 2.30 \pm 0.3$ ) and was no longer apparent at an ISI of $200 \mathrm{~ms}\left(\mathrm{PPR}_{200 \mathrm{~ms}}, 1.05 \pm 0.08\right)$. The decay of facilitation was fit by a single exponential with a time constant of $14 \mathrm{~ms}$, indicating that NCS-1 is a potent and fast-acting regulator of short-term plasticity (Fig. 2b). The observed effects of NCS-1 on short-term plasticity could result from either a reduction in PPD or an enhancement of PPF. To address this issue, we carried out experiments in low extracellular calcium $(1.0 \mathrm{mM})$. This relieved depression and allowed for slight facilitation in non-transfected cells (Fig. 2c, open circles; $P_{P R} 20 \mathrm{~ms}, 1.25 \pm$ $0.06 ; \mathrm{PPR}_{50 \mathrm{~ms}}, 1.12 \pm 0.04 ; \mathrm{PPR}_{200 \mathrm{~ms}}, 1.04 \pm 0.01$ ). Consistent with an enhancement of PPF by NCS-1, transfected neurons in low calcium showed more facilitation than did control cells at all ISIs tested (Fig. 2c, filled triangles; PPR $20 \mathrm{~ms}, 2.32 \pm 0.13$; $\mathrm{PPR}_{50 \mathrm{~ms}}, 1.61 \pm 0.08$; $\mathrm{PPR}_{200 \mathrm{~ms}}, 1.26 \pm 0.07$ ).

To confirm that the effect of NCS-1 depends on its ability to bind calcium, we expressed a mutant form of NCS-1 with a single amino acid change at position 120 (E120Q) in one of the three functional calcium-binding EF-domains ${ }^{22}$. E120Q had a much reduced activity compared to wildtype NCS-1 (PPR $50 \mathrm{~ms}, 0.97 \pm 0.10$; $\mathrm{PPR}_{100 \mathrm{~ms}}, 0.87 \pm 0.11 ; n=8, P<0.05$; data not shown). The residual activity of E120Q indicates that the mutant protein still folds correctly and that alterations to one of its calcium binding properties sites reduces, but does not eliminate ${ }^{23}$, calcium binding activity.

In neuroendocrine cells, NCS-1 interacts with phosphatidylinositol-4-kinase (PI4K) as its downstream target, and this interaction depends on the myristoylated amino-terminus of NCS-1 (ref. 23). To test whether myristoylation, and thus PI4K activation, is necessary for NCS-1 activity in neurons, we transfected hippocampal cultures with a point mutant of NCS-1 that cannot be myristoylated. This mutant was able to switch depression to 
facilitation and was in fact indistinguishable from wild-type NCS-1 in its effect on shortterm facilitation $\left(\mathrm{PPR}_{50 \mathrm{~ms}}, 1.21 \pm 0.15 ; \mathrm{PPR}_{100 \mathrm{~ms}}, 1.16 \pm 0.08 ; n=8, P>0.1\right)$. We conclude that in neurons, NCS-1 does not need to activate PI $4 \mathrm{~K}$, but rather acts directly as a calcium sensor for short-term facilitation through calcium-dependent interactions with other proteins ${ }^{24}$.

As short-term facilitation often inversely correlates with initial release probability, it was important to determine whether the amplitude of the first response was affected by NCS-1. In the group data (Fig. 1f) and across experiments (Fig. 2d), transfection of neurons with NCS-1 did not change the amplitude of the synaptic response (Fig. 2d; control, $187 \pm 44 \mathrm{pA}$, $n=10$; NCS-1, $193 \pm 52 \mathrm{pA}, n=10$; E120Q, $185 \pm 66 \mathrm{pA}, n=8)$. Thus, baseline neurotransmitter release was not affected in NCS-1 transfected neurons, indicating that basal synaptic strength and short-term plasticity can be independently regulated (see below).

Immunocytochemical labeling of transfected hippocampal neurons with an antibody ${ }^{25}$ against NCS-1 confirmed that GFP-expressing neurons had higher levels of NCS-1 than non-transfected control neurons in the same dish (Fig. 3a-c). In fact, non-transfected cultured hippocampal neurons showed only background levels of NCS-1 at amounts comparable to those found in glial cells (Fig. 3c). Given that NCS-1 is highly expressed in hippocampal neurons in vivo ${ }^{25}$, it may be that in culture, these neurons downregulate NCS-1 levels, although it is difficult to compare in vivo and in vitro levels of proteins. However, NCS-1 was markedly downregulated in neurons that were subjected to the calciumphosphate transfection protocol compared to non-treated 'naive' neurons (Fig. 3d,e). Thus, we compared paired-pulse plasticity in naive neurons (Fig. 3d,f, hatched bars) with plasticity in neurons that had been treated with calcium phosphate but not transfected with any construct (Fig. 3e,f, open bars). Consistent with our findings that increased NCS-1 enhances facilitation, neurons with decreased levels of NCS-1 showed less facilitation, manifested as increased depression, as compared to control (Fig. 3d).

\section{NCS-1 facilitates transmission during high-frequency trains}

To test the robustness of NCS-1-induced enhancement of neurotransmitter release, we expanded the pairs of pulses to trains of ten stimuli with varying frequencies $(20,33,50 \mathrm{~Hz})$, followed by a test stimulus $200 \mathrm{~ms}$ after the train. In control neurons, at all frequencies tested, depression quickly saturated after the second or third stimulus at $~ 50 \%$ of baseline (Fig. 4a,b). Depression did not fully recover during the $200 \mathrm{~ms}$ before the test pulse, indicating that releasable vesicles cannot be easily made available during this time interval ${ }^{5}$.

In contrast, at NCS-1-transfected synapses, facilitation evident after the second stimulus was maintained throughout the train (Fig. 4a,c-e). At higher stimulation frequencies (20 and 30 ms ISI), maximal facilitation of approximately $3 \times$ the initial response was reached after the second stimulus ( $266 \pm 64 \%$ at $20 \mathrm{~ms}$ ISI, Fig. $3 \mathrm{c} ; 324 \pm 74 \%$ at $30 \mathrm{~ms}$ ISI, Fig. $4 \mathrm{~d}$ ), but at lower frequencies (50 ms ISI), peak facilitation was not reached until the fourth or fifth stimulus ( $297 \pm 77 \%$; Fig. 4 d). The decrement after the peak response shows that NCS-1 allows for facilitation on top of slow depression. This is consistent with our interpretation that NCS-1 acts primarily on facilitation rather than on depression. At 20 and $30 \mathrm{~ms}$ ISI, facilitation decreased after the fourth stimulus, but the test stimulus at $200 \mathrm{~ms}$ showed a surprisingly strong rebound (Fig. $4 \mathrm{c}, \mathrm{d}$ ). The persistence of facilitation during stimulus trains illustrates the ability of NCS-1 to enhance and sustain neurotransmitter release, especially at high frequencies. To test whether asynchronous release was enhanced after NCS-1 transfection, we measured the integral of the current after the last stimulus of the train and before the test pulse ${ }^{26}$. Consistent with a higher rate of release, the integrated charge, and thus asynchronous release, was significantly enhanced after NCS-1 transfection (Fig. 4b; control, $1.04 \pm 0.28 \mathrm{pC}, n=7$; NCS-1, $3.28 \pm 0.79 \mathrm{pC}, n=6 ; P<0.05)$. 


\section{NCS-1 does not alter calcium currents}

NCS-1 has been reported to enhance calcium influx at the calyx of $\mathrm{Held}^{18}$, whereas in PC12 and chromaffin cells, NCS-1 inhibits calcium channels ${ }^{27}$.We thus tested whether NCS-1 alters the amplitude and/or activation kinetics of non-L-type calcium channels known to mediate neurotransmitter release in hippocampal neurons ${ }^{28}$. The calcium currents in transfected versus non-transfected cells were indistinguishable at all voltages tested (Fig. $5 a)$, resulting in overlapping current-voltage $(I-V)$ curves (Fig. 5b). No difference in calcium-channel activation kinetics was observed (Fig. 5c). Similarly, paired-pulse stimulation induced considerable depression of the calcium current amplitude with no change in activation kinetics in control or NCS-1 transfected neurons (Fig. 5d). The depression in calcium currents might explain some of the synaptic PPD observed at control synapses, but it is incongruent with the synaptic facilitation observed in NCS-1 transfected neurons. We conclude that in hippocampal neurons, NCS-1 does not exert its effect through altered calcium currents.

\section{NCS-1 does not change basal release probability}

In many systems, paired-pulse plasticity strongly correlates with release probability ${ }^{4}$, thus NCS-1 could be lowering release probability, allowing for the switch from depression to facilitation. We measured release probability using the non-competitive NMDA-receptor antagonist MK-801. MK-801 irreversibly blocks NMDA channels only when they are open $^{29}$, so that the rate of block during low frequency stimulation is proportional to the release probability ${ }^{30,31}$. NMDA receptor-mediated EPSCs showed no difference in amplitude between transfected and non-transfected cells (Fig. 6a, inset; control, $139 \pm 25, n$ $=6$; NCS-1, $145 \pm 28, n=7, P>0.8$ ). Surprisingly, the rate of block between control neurons and NCS-1-transfected neurons was identical, indicating that basal release probability was not changed by NCS-1 (Fig. 6a; $n=7$ for both groups; $P=0.62$ for pulses $1-20 ; F_{1,19}=0.241$, two-way ANOVA).

To ensure that our methods could detect changes in release probability, we lowered release probability by lowering the extracellular calcium concentration from $2 \mathrm{mM}$ to $1 \mathrm{mM}$. This markedly reduced the amplitude of the EPSC $\left(1 \mathrm{mM} \mathrm{Ca}^{2+}, 68.6 \pm 28 \mathrm{pA}, n=8 ; 2 \mathrm{mM} \mathrm{Ca}^{2+}\right.$, $187 \pm 44 \mathrm{pA} ; n=10 ; P<0.05)$ and, consistent with a lower release probability, PPD was converted to a slight potentiation (Fig. 6b, inset; PPR in $1 \mathrm{mM} \mathrm{Ca}^{2+}, 1.14 \pm 0.07$; PPR in 2 $\left.\mathrm{mM} \mathrm{Ca}^{2+}, 0.56 \pm 0.09\right)$. As expected, MK801 block was significantly slower in low $\mathrm{Ca}^{2+}$ (Fig. $6 \mathrm{~b} ; n=7$ for both groups; $P<0.001$ for pulses $1-20 ; F_{1,19}=309.1$, two-way ANOVA). This demonstrates that our method was sensitive enough to detect changes in release probability of the magnitude needed to explain our data and supports the conclusion that NCS-1 does not alter basal release probability.

Although NCS-1 does not alter release probability during the first stimulus, it might selectively increase the probability of release during the second stimulus. To test this, we repeated the MK-801 experiments using pairs of stimuli (50 ms ISI). In contrast to the single-pulse experiments, the rate of MK- 801 block was faster in NCS-1 transfected neurons than in control neurons (Fig. $6 \mathrm{c} ; n=5$ for both groups; $P<0.001$ for pulses $1-20 ; F_{1,19}=$ 82.3, two-way ANOVA). Taken together, these results indicate that the paired-pulse facilitation we observed in NCS-1 neurons resulted from an increase in the probability of release during the second stimulus but not the first.

\section{NCS-1 expression confers more reliable postsynaptic firing}

Increased neurotransmitter output could allow postsynaptic neurons to reach threshold more consistently during repetitive stimulation. To test this prediction we recorded synaptic transmission between pairs of neurons in current clamp mode. In agreement with our 
voltage-clamp data (Figs. 2 and 3), paired-pulse stimulation of control neurons (Fig. 7b) resulted in depression of the excitatory postsynaptic potential (EPSP). However, pairedpulse stimulation of transfected neurons (Fig. 7a) resulted in facilitation of the EPSP, which brought the postsynaptic neuron to threshold. When control neurons were stimulated with a train of stimuli, the EPSPs depressed in response to successive stimuli (Fig. 7c). Stimulation of the NCS-1-transfected neuron with a train of the same frequency resulted in facilitation of the EPSP that consistently brought the postsynaptic cell to threshold (Fig. 7d). Thus, NCS-1induced presynaptic facilitation allows postsynaptic neurons to fire action potentials more readily in response to repetitive stimulation, increasing their activity within neuronal networks.

\section{DISCUSSION}

We have shown that dissociated hippocampal neurons can form synaptic connections with differing characteristics of short-term plasticity, depending on the expression levels of the calcium-binding protein NCS-1. Our results provide strong evidence that the molecular makeup of a synapse can allow neurons to acutely change their short-term plasticity characteristics. NCS-1 enhances neurotransmitter release during pairs of stimuli and stimulus trains, but it does not alter basal release probability. Thus, the magnitude and direction of short-term plasticity is not merely a result of establishing a synaptic connection but can be set independently of basal neurotransmitter output.

NCS-1 has previously been shown to be involved in different systems, but until now it has remained untested whether it has a distinct effect on synaptic transmitter release in the mammalian CNS. Our findings suggest that the function of NCS- 1 on synaptic transmission in the hippocampus is quite different from its documented role in other systems. Unlike the findings at the calyx of Held and in chromaffin cells, we did not observe an alteration of calcium current characteristics (Fig. 5). In chromaffin and PC-12 cells, NCS-1 has been shown to have a regulatory role on ATP-evoked, but not depolarization-triggered, release $^{32,33}$. At Drosophila neuromuscular junctions, overexpressing the NCS-1 homolog frequenin enhanced neurotransmitter release by eliciting 'large facilitated responses,' but this action is thought to be mediated through indirect mechanisms rather than directly through an effect on release ${ }^{15,34}$. In contrast, our data show that NCS-1 directly affects neurotransmitter release, acting as a calcium sensor for short-term plasticity.

Several models have been proposed to account for short-term facilitation, most of which assume that facilitation depends on a buildup of 'residual' calcium that persists after previous stimulations ${ }^{9}$. Consistent with this interpretation, slow calcium buffers have been shown to affect short-term facilitation at many synapses (for review, see refs. 4,14). Experiments at the calyx of Held show that summation of residual calcium with the newly entering calcium can almost completely account for facilitation ${ }^{10}$. However, in many systems, residual calcium levels in the presynaptic terminal seem too low to have a substantial summating effect ${ }^{14}$ and/or decay more rapidly than facilitation ${ }^{13}$. An alternate model that has recently been validated for a subset of synapses (but explicitly not the hippocampal CA3-CA1 synapse) proposes that saturation of local calcium buffers can enhance release and thus cause facilitation ${ }^{12}$. As NCS-1 binds calcium in the hundreds-ofnanomolar to low-micromolar range ${ }^{35}$, it could conceivably act as a calcium buffer altering calcium-dependent intracellular processes. However, if NCS-1 were promoting facilitation by acting as a fast, saturable buffer in promoting facilitation, it should be present at levels that affect basal transmission ${ }^{36}$, which is opposite to our findings (Figs. $2 \mathrm{f}$ and 4 ). Moreover, lowering extracellular calcium would be expected to decrease buffer saturation and thus decrease facilitation ${ }^{12}$, which again is in contrast to our findings. Could NCS-1 instead act as a slow buffer to alter intracellular calcium signaling? Recordings done in hippocampal 
cultures in the presence of the slow membrane-permeable calcium buffer EGTA-AM show no effect of weakly buffering calcium on either EPSC amplitude or PPD, whereas higher buffer concentrations reportedly do affect basal transmission ${ }^{26}$. Furthermore, application of EGTA-AM to acute hippocampal slices blocked, rather than mediated, paired-pulse facilitation ${ }^{12}$. In summary, our experiments are inconsistent with a major role of NCS-1 as a calcium buffer. Therefore, we propose that NCS-1 primarily acts as a calcium sensor for short-term plasticity.

Buffering of calcium by either slow or fast buffers does not account for all forms of facilitation. Thus, a third model proposes that distinct presynaptic calcium binding sites mediate facilitation and act in cooperation with the main release sensor ${ }^{13,37}$, but the molecular identity of that sensor has remained elusive ${ }^{4}$. Our data now strongly suggest that NCS-1 acts as such a calcium sensor for short-term facilitation. In normal extracellular calcium, expression of NCS-1 switches synaptic plasticity from depression to facilitation, and reducing the ability of NCS-1 to bind calcium (E120Q mutant) reduces this effect. How can we distinguish between NCS-1 acting as a calcium sensor that alleviates depression versus one that enhances facilitation? Reducing extracellular calcium showed that even when depression is alleviated, NCS-1 further enhances facilitation (Fig. 2c). Furthermore, in stimulus trains, NCS-1 allows for facilitation on top of slow depression and enhances desynchronized release (Fig. 4b,c). Taken together, these data demonstrate that NCS-1 enhances facilitation, rather than alleviating depression, and support the idea that occupation of this protein's calcium binding sites is crucial for the expression of short-term facilitation.

In the present study, we show that altered expression levels of NCS-1 can strongly regulate release dynamics in individual neurons. Hippocampal neurons in vivo 4 show robust facilitation and relatively high levels of NCS-1 (ref. 25). In contrast, transfected hippocampal neurons in culture show only limited expression of NCS-1 (Fig. 1) and pairedpulse depression (Figs. 2-4,6,7). This phenotype can be reverted by NCS-1 transfection (Figs. 1-4,6,7). Likewise, differences in short-term plasticity in vivo may, in part, be caused by differing levels of NCS-1 expression. This interpretation is supported by the finding that hippocampal mossy fiber inputs onto CA3 pyramidal cells show stronger facilitation than seen in associative/commissural synapses ${ }^{38}$ and have higher expression levels of NCS-1 (ref. 25). Intriguingly, neurons can also dynamically change expression levels of NCS-1, as has been shown after LTP induction ${ }^{39}$ and in patients with schizophrenia ${ }^{40}$. In view of the present data, we propose that neurons can alter their contribution to neuronal networks by dynamically controlling their short-term plasticity characteristics via differential and regulated expression of NCS-1.

\section{METHODS}

\section{Primary cell cultures}

Rat hippocampal neurons were cultured as previously described ${ }^{41}$ with slight modifications in accordance with the protocol approved by the UCLA Chancellor's Animal Research Committee. The hippocampal CA3-CA1 regions were dissected from postnatal day 0-2 Sprague Dawley rat brains in Hank's balanced salt solution (HBSS, Life Technologies) containing 20\% fetal bovine serum (FBS, Hyclone). The tissue was digested for 5 min with $20 \mathrm{mg} / \mathrm{ml}$ of trypsin, then cells were freed by trituration and plated on Matrigel (Life Technologies) coated glass coverslips at a density of 15,000-30,000 cells $/ \mathrm{cm}^{2}$ inside an 8$\mathrm{mm}$ diameter glass ring. Cells were grown in minimum essential medium (MEM, Life Technologies) supplemented with $0.5 \%$ glucose, $100 \mathrm{mg} / \mathrm{l}$ bovine transferrin (Calbiochem), $24 \mathrm{mg} / \mathrm{l}$ insulin, $2 \mathrm{mM}$ GlutaMax-1 (Life Technologies) and 10\% FBS (Hyclone) in 5\% $\mathrm{CO}_{2}$ atmosphere at $37{ }^{\circ} \mathrm{C}$. Cells were fed after 1-2 days in vitro (d.i.v.) with supplemented MEM 
containing 5\% FBS, 2\% B-27 (Gibco) and $5 \mu \mathrm{M}$ Cytosine arabinoside to inhibit glial proliferation. Unless otherwise stated, all chemicals were obtained from Fluka or Sigma.

\section{Transfection}

NCS-1 and the E120Q mutant were cloned into the BamHI/XhoI site of the bi-cistronic pIRES2 hrGFP 1A vector (Stratagene), and plasmid DNA was purified using an endotoxinfree maxi-prep kit (Qiagen). The bi-cistronic pIRES2 hrGFP 1A vector directs expression of NCS-1 or the E120Q mutant and GFP from an internal ribosomal entry site (IRES).

Neurons were transfected using calcium phosphate-mediated gene transfer ${ }^{42}$ after cells were 7-8 d.i.v. We used $9 \mu \mathrm{g} / \mathrm{ml}$ of DNA and left the calcium/DNA precipitate to form in the dark at room temperature for $15 \mathrm{~min}$. Neurons were exposed to the precipitate for 20-25 min in Opti-MEM (Gibco-BRL) in the presence of $10 \mu \mathrm{M}$ CNQX. Coverslips were subsequently washed thoroughly with Opti-MEM and then returned to the original culture media until use. Transfection efficiency was typically between 5 and $10 \%$, with no apparent toxicity to the cells.

For immunohistochemistry, cells were fixed with $4 \%$ paraformaldehyde in PBS, permeabilized with $0.1 \%$ Triton X-100 in PBS, blocked with $2 \%$ goat serum in PBS and incubated overnight at $4{ }^{\circ} \mathrm{C}$ with primary antibodies (1:100 affinity-purified rabbit antiNCS-1 (ref. 25) and 1:1,000 chicken anti-GFP, AB16901 from Chemicon).After washing, cells were incubated for $1 \mathrm{~h}$ at room temperature in secondary antibody (1:400 Alexa Flour 488 donkey-anti-rabbit, and 1:400 Alexa Flour 546 goat-anti-chicken, Molecular Probes), washed and mounted with Immuno Floure Mounting Medium (ICN Biomedicals, \#622701). Images were obtained on a Zeiss LM410 confocal microscope.

\section{Electrophysiology}

Whole-cell patch-clamp recordings from transfected and nontransfected hippocampal neurons were performed at room temperature $\left(20-22{ }^{\circ} \mathrm{C}\right) 1-4 \mathrm{~d}$ after transfection (9-12 d.i.v.). Coverslips were mounted in a perfusion chamber on a compound microscope (Zeiss) and standard epifluorescence was used to identify transfected cells based on the expression of GFP. The recording chamber was perfused with artificial cerebral spinal fluid (ACSF) containing $119 \mathrm{mM} \mathrm{NaCl}, 5 \mathrm{mM} \mathrm{KCL}, 30 \mathrm{mM}$ glucose, $25 \mathrm{mM}$ HEPES, $2 \mathrm{mM} \mathrm{CaCl}_{2}, 2 \mathrm{mM}$ $\mathrm{MgCl}_{2}$ and $0.01 \mathrm{mM}$ glycine ( $\mathrm{pH} 7.3 ; 310$ mosm). Electrodes were pulled from borosilicate glass capillaries (Warner) to a final tip resistance of 4-7 M $\Omega$. The electrode solution consisted of $60 \mathrm{mM} \mathrm{KCl}, 40 \mathrm{mM}$ methanesulfonic acid, $30 \mathrm{mM}$ HEPES, $10 \mathrm{mM}$ sodium phosphocreatine, $5 \mathrm{mM} \mathrm{MgCl} 2,5 \mathrm{mM}$ ATP, $0.6 \mathrm{mM}$ EGTA and $0.3 \mathrm{mM}$ GTP (pH 7.3; 295 mosm). Neurons were voltage-clamped at $-70 \mathrm{mV}$ using Axopatch $200 \mathrm{~A}$ (Axon Instruments) and Cairn Optopatch (Cairn Research Ltd, Faversham, Kent, UK) patch clamp amplifiers. Membrane currents were low-pass filtered at $10 \mathrm{kHz}$ and acquired at $50 \mathrm{kHz}$ using a computer interface (6502E, National Instruments). Software was written in LabView (National Instruments, written by F.E.S. and Z. Zhou). Pairs of neurons were each voltageclamped at $-70 \mathrm{mV}$, close to the resting membrane potential (the holding current was $<100$ pA in all experiments). Series resistance of 10-25 M $\Omega$ was not compensated, and experiments were terminated if significant variation $(>25 \%)$ occurred during the experiment. To measure synaptic transmission between pairs of voltage-clamped neurons, one neuron (presynaptic) was briefly depolarized (1 ms) to $+50 \mathrm{mV}$ to elicit an escape action potential. The response was recorded in the other (postsynaptic neuron) as an excitatory inward current. Most pairs had mono-synaptic connections in both directions, and frequently an autaptic response could also be seen in the presynaptic neuron. This allowed for internal controls in the very same recording (Figs. 1,2). Single stimuli were delivered every 15-30 s with no obvious change in response amplitude during the recording (Fig. 1). 
For measurements of release probability (Fig. 5) using the non-competitive NMDA antagonist MK801,NMDA receptor-mediated currents were isolated by adding $5 \mu \mathrm{M}$ CNQX disodium salt and $100 \mu \mathrm{M}$ picrotoxin to, and removing magnesium from, the bath solution. Recordings were typically done 3-4 d after transfection.

Calcium currents were recorded from single neurons using a $\mathrm{P} / 4$ protocol implemented in JClamp (SciSoft). The internal solution contained $30 \mathrm{mM} \mathrm{CsCl,} 20 \mathrm{mM}$ TEA Cl, $50 \mathrm{mM}$ cesium methane sulfonate, $0.6 \mathrm{mM}$ EGTA, $30 \mathrm{mM}$ HEPES, $5 \mathrm{mM} \mathrm{MgCl}_{2}, 5 \mathrm{mM} \mathrm{Na}_{2} \mathrm{ATP}$, $10 \mathrm{mM}$ sodium phosphocreatine, $0.3 \mathrm{mM} \mathrm{NaGTP}$ (pH 7.2, $295 \mathrm{mosm}$ ). To isolate calcium currents that underlie synaptic transmission in hippocampal neurons ( $\mathrm{N}$ and P/Q-type ${ }^{28}$, we included in the external solution $1 \mu \mathrm{M}$ TTX to block sodium channels and $3 \mu \mathrm{M}$ nimodipine to block L-type calcium channels. Voltages were corrected for series resistance errors, and analysis was done using JClamp software. Calcium current $I-V$ curves were obtained by stepping the cell from the holding potential to a test potential for $100 \mathrm{~ms}$ every $5 \mathrm{~s}$. The amplitude at the end of the voltage step was normalized for the cell membrane capacitance and plotted against the test potential. To determine effects of short-term plasticity on calcium currents, two 20-ms depolarizations to $+10 \mathrm{mV}$ were given with varying ISIs (10$80 \mathrm{~ms})$.

For current clamp recordings (Fig. 6), both neurons were kept at resting potentials of -75 to $-80 \mathrm{mV}$ by injecting a small bias current. Action potentials were triggered by injecting a depolarizing current for $5 \mathrm{~ms}$.

Data were additionally analyzed and figures were made using Origin (Originlab). Data are expressed as mean \pm s.e.m. and, unless otherwise indicated, the Student's $t$-test was used to determine significance $(P<0.05) . n$ refers to the number of individual neuronal pairs tested.

\section{Acknowledgments}

We thank B. Hargrove for technical assistance and all members of the Schweizer lab. We are also grateful to D. Buonomano, A. Dempsey, J.L. Feldman, M. Klein, N.A. Lambert, K.C. Martin, T.S. Otis, T.J. O’Dell, S.L. Smith and S.A. White for valuable and encouraging discussions, and H.T. Blair for help with statistics. This work was supported by grants from the Whitehall Foundation and the National Institutes of Health (NS41317) to F.E.S.

\section{References}

1. Tsodyks MV, Markram H. The neural code between neocortical pyramidal neurons depends on neurotransmitter release probability. Proc. Natl. Acad. Sci. USA. 1997; 94:719-723. [PubMed: 9012851]

2. Buonomano DV. Decoding temporal information: a model based on short-term synaptic plasticity. J. Neurosci. 2000; 20:1129-1141. [PubMed: 10648718]

3. Marder E. From biophysics to models of network function. Annu. Rev. Neurosci. 1998; 21:25-45. [PubMed: 9530490]

4. Atwood HL, Karunanithi S. Diversification of synaptic strength: presynaptic elements. Nat. Rev. Neurosci. 2002; 3:497-516. [PubMed: 12094207]

5. Stevens CF, Tsujimoto T. Estimates for the pool size of releasable quanta at a single central synapse and for the time required to refill the pool. Proc. Natl. Acad. Sci. USA. 1995; 92:846-849.

[PubMed: 7846064]

6. Rosenmund C, Stevens CF. Definition of the readily releasable pool of vesicles at hippocampal synapses. Neuron. 1996; 16:1197-1207. [PubMed: 8663996]

7. Waldeck RF, Pereda A, Faber DS. Properties and plasticity of paired-pulse depression at a central synapse. J. Neurosci. 2000; 20:5312-5320. [PubMed: 10884315]

8. Zucker RS. Calcium- and activity-dependent synaptic plasticity. Curr. Opin. Neurobiol. 1999; 9:305-313. [PubMed: 10395573] 
9. Katz B, Miledi R. The role of calcium in neuromuscular facilitation. J. Physiol. (Lond.). 1968; 195:481-492. [PubMed: 4296699]

10. Felmy F, Neher E, Schneggenburger R. Probing the Intracellular Calcium Sensitivity of Transmitter Release during Synaptic Facilitation. Neuron. 2003; 37:801-811. [PubMed: 12628170]

11. Rozov A, Burnashev N, Sakmann B, Neher E. Transmitter release modulation by intracellular $\mathrm{Ca}^{2+}$ buffers in facilitating and depressing nerve terminals of pyramidal cells in layer $2 / 3$ of the rat neocortex indicates a target cell-specific difference in presynaptic calcium dynamics. J. Physiol. 2001; 531:807-826. [PubMed: 11251060]

12. Blatow M, Caputi A, Burnashev N, Monyer H, Rozov A. $\mathrm{Ca}^{2+}$ buffer saturation underlies paired pulse facilitation in calbindin-d28k-containing terminals. Neuron. 2003; 38:79-88. [PubMed: 12691666]

13. Atluri PP, Regehr WG. Determinants of the time course of facilitation at the granule cell to Purkinje cell synapse. J. Neurosci. 1996; 16:5661-5671. [PubMed: 8795622]

14. Zucker RS, Regehr WG. Short-term synaptic plasticity. Annu. Rev. Physiol. 2002; 64:355-405. [PubMed: 11826273]

15. Pongs O, et al. Frequenin: a novel calcium-binding protein that modulates synaptic efficacy in the Drosophila nervous system. Neuron. 1993; 11:15-28. [PubMed: 8101711]

16. Burgoyne RD, Weiss JL. The neuronal calcium sensor family of $\mathrm{Ca}^{2+}$ binding proteins. Biochem. J. 2001; 353:1-12. [PubMed: 11115393]

17. Olafsson P, Wang T, Lu B. Molecular cloning and functional characterization of the XenopusCa ${ }^{2+}$ binding protein frequenin. Proc. Natl. Acad. Sci. USA. 1995; 92:8001-8005. [PubMed: 7644528]

18. Tsujimoto T, Jeromin A, Saitoh N, Roder JC, Takahashi T. Neuronal calcium sensor 1 and activity-dependent facilitation of P/Q-type calcium currents at presynaptic nerve terminals. Science. 2002; 295:2276-2279. [PubMed: 11910115]

19. Tong G, Jahr CE. Multivesicular release from excitatory synapses of cultured hippocampal neurons. Neuron. 1994; 12:51-59. [PubMed: 7507341]

20. Wilcox KS, Dichter MA. Paired pulse depression in cultured hippocampal neurons is due to a

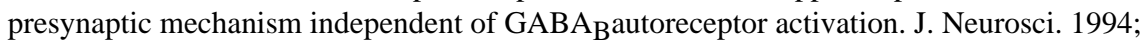
14:1775-1788. [PubMed: 8126570]

21. Debanne D, Geahwiler BH, Thompson SM. Long-term synaptic plasticity between pairs of individual CA3 pyramidal cells in rat hippocampal slice cultures. J. Physiol. (Lond.). 1998; 507:237-247. [PubMed: 9490845]

22. Weiss JL, Archer DA, Burgoyne RD. Neuronal $\mathrm{Ca}^{2+}$ sensor-1/frequenin functions in an autocrine pathway regulating $\mathrm{Ca}^{2+}$ channels in bovine adrenal chromaffin cells. J. Biol. Chem. 2000; 275:40082-40087. [PubMed: 11006299]

23. Rajebhosale M, Greenwood S, Vidugiriene J, Jeromin A, Hilfiker S. Phosphatidylinositol 4-OH kinase is a downstream target of neuronal calcium sensor-1 in enhancing exocytosis in neuroendocrine cells. J. Biol. Chem. 2003; 278:6075-6084. [PubMed: 12471042]

24. McFerran BW, Weiss JL, Burgoyne RD. Neuronal $\mathrm{Ca}^{2+}$ sensor 1 . Characterization of the myristoylated protein, its cellular effects in permeabilized adrenal chromaffin cells, $\mathrm{Ca}^{2+}$ independent membrane association, and interaction with binding proteins, suggesting a role in rapid $\mathrm{Ca}^{2+}$ signal transduction. J. Biol. Chem. 1999; 274:30258-30265. [PubMed: 10514519]

25. Jinno S, Jeromin A, Roder J, Kosaka T. Immunocytochemical localization of neuronal calcium sensor-1 in the hippocampus and cerebellum of the mouse, with special reference to presynaptic terminals. Neuroscience. 2002; 113:449-461. [PubMed: 12127101]

26. Cummings DD, Wilcox KS, Dichter MA. Calcium-dependent paired-pulse facilitation of miniature EPSC frequency accompanies depression of EPSCs at hippocampal synapses in culture. J. Neurosci. 1996; 16:5312-5323. [PubMed: 8757244]

27. Weiss JL, Burgoyne RD. Voltage-independent inhibition of P/Q-type $\mathrm{Ca}^{2+}$ channels in adrenal chromaffin cells via a neuronal $\mathrm{Ca}^{2+}$ sensor-1-dependent pathway involves Src family tyrosine kinase. J. Biol. Chem. 2001; 276:44804-44811. [PubMed: 11583988] 
28. Wheeler DB, Randall A, Tsien RW. Roles of N-type and Q-type $\mathrm{Ca}^{2+}$ channels in supporting hippocampal synaptic transmission. Science. 1994; 264:107-111. [PubMed: 7832825]

29. Huettner JE, Bean BP. Block of Nmethyldaspartate-activated current by the anticonvulsant MK-801: selective binding to open channels. Proc. Natl. Acad. Sci. USA. 1988; 85:1307-1311. [PubMed: 2448800]

30. Hessler NA, Shirke AM, Malinow R. The probability of transmitter release at a mammalian central synapse. Nature. 1993; 366:569-572. [PubMed: 7902955]

31. Rosenmund C, Clements JD, Westbrook GL. Nonuniform probability of glutamate release at a hippocampal synapse. Science. 1993; 262:754-757. [PubMed: 7901909]

32. McFerran BW, Graham ME, Burgoyne RD. Neuronal $\mathrm{Ca}^{2+}$ sensor 1, the mammalian homologue of frequenin, is expressed in chromaffin and PC12 cells and regulates neurosecretion from dense-core granules. J. Biol. Chem. 1998; 273:22768-22772. [PubMed: 9712909]

33. Koizumi S, et al. Mechanisms underlying the neuronal calcium sensor-1-evoked enhancement of exocytosis in PC12 cells. J. Biol. Chem. 2002; 277:30315-30324. [PubMed: 12034721]

34. Rivosecchi R, Pongs O, Theil T, Mallart A. Implication of frequenin in the facilitation of transmitter release in Drosophila. J. Physiol. 1994; 474:223-232. [PubMed: 7911829]

35. Cox JA, et al. Cation binding and conformational changes in VILIP and NCS-1, two neuronspecific calcium-binding proteins. J. Biol. Chem. 1994; 269:32807-32813. [PubMed: 7806504]

36. Zucker RS. Increased $\mathrm{Ca}^{2+}$ buffering enhances $\mathrm{Ca}^{2+}$ dependent process. J. Physiol. 2001; 531:583. [PubMed: 11251041]

37. Vyshedskiy A, Allana T, Lin JW. Analysis of presynaptic $\mathrm{Ca}^{2+}$ influx and transmitter release kinetics during facilitation at the inhibitor of the crayfish neuromuscular junction. J. Neurosci. 2000; 20:6326-6332. [PubMed: 10964937]

38. Salin PA, Scanziani M, Malenka RC, Nicoll RA. Distinct short-term plasticity at two excitatory synapses in the hippocampus. Proc. Natl. Acad. Sci. USA. 1996; 93:13304-13309. [PubMed: 8917586]

39. Genin A, et al. Regulated expression of the neuronal calcium sensor-1 gene during long-term potentiation in the dentate gyrus in vivo. Neuroscience. 2001; 106:571-577. [PubMed: 11591457]

40. Koh PO, et al. Up-regulation of neuronal calcium sensor-1 (NCS-1) in the prefrontal cortex of schizophrenic and bipolar patients. Proc. Natl. Acad. Sci. USA. 2003; 100:313-317. [PubMed: 12496348]

41. Ryan TA, et al. The kinetics of synaptic vesicle recycling measured at single presynaptic boutons. Neuron. 1993; 11:713-724. [PubMed: 8398156]

42. Threadgill R, Bobb K, Ghosh A. Regulation of dendritic growth and remodeling by Rho, Rac and Cdc42. Neuron. 1997; 19:625-634. [PubMed: 9331353] 

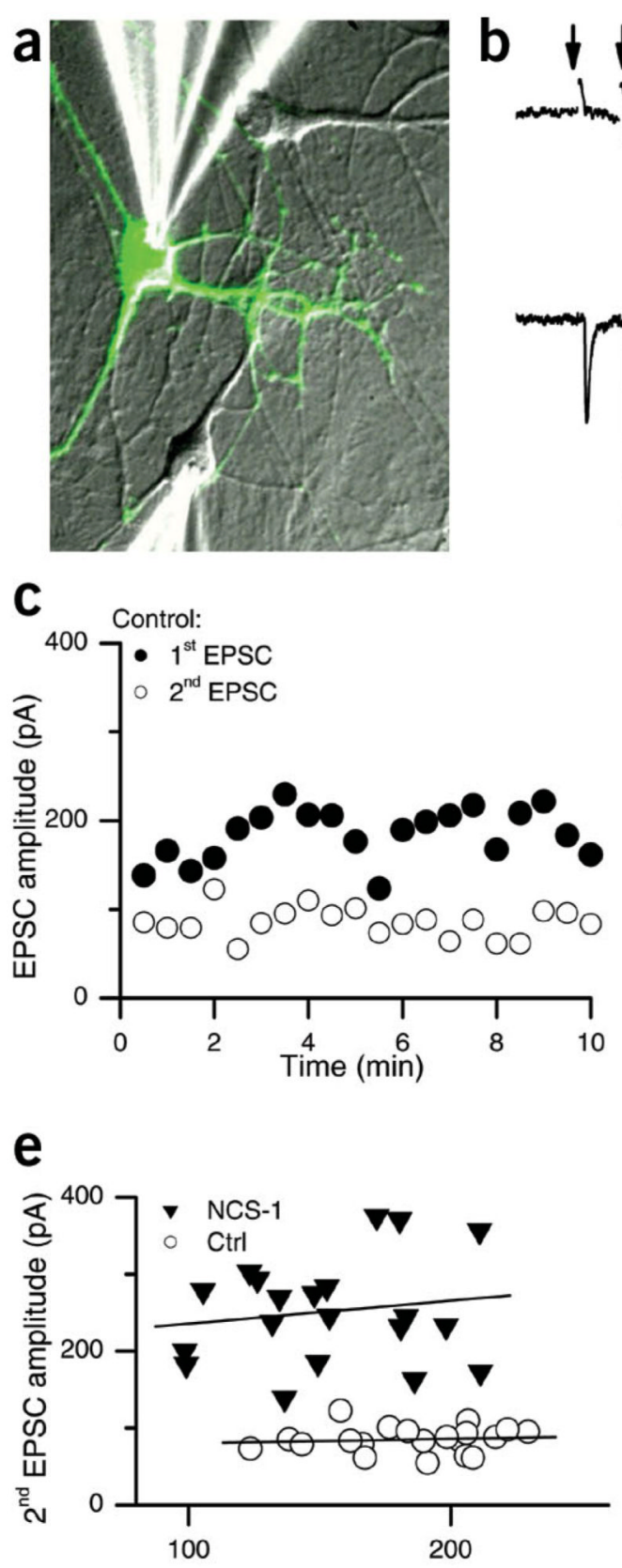

First EPSC Amplitude (pA)
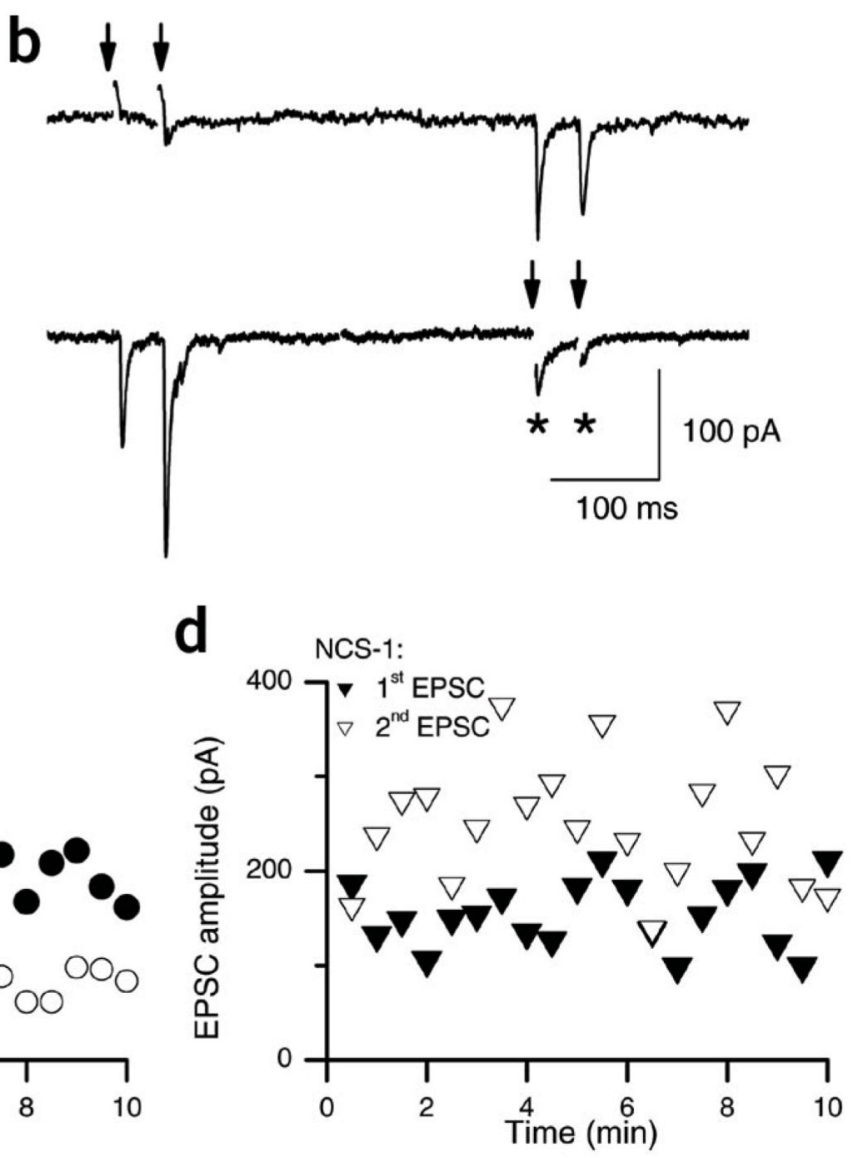

f

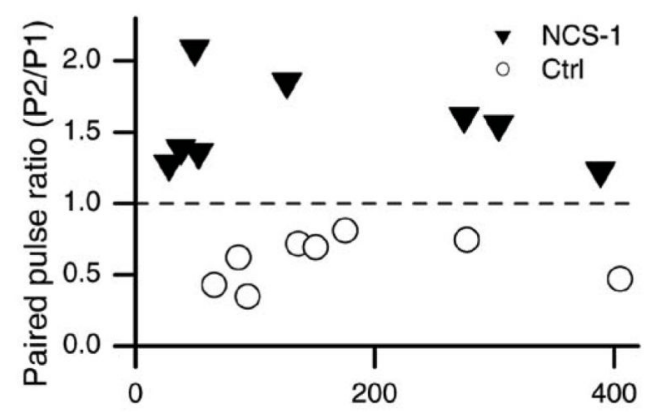

First EPSC amplitude (pA)

Figure 1.

Pair recordings from reciprocally connected neurons reveal a switch from paired-pulse depression to facilitation at NCS-1-transfected synapses. (a) Superposition of fluorescence (green) and transmitted light images (gray) of cultured hippocampal neurons at 9 d.i.v. and 2 $\mathrm{d}$ after transfection with NCS-1. The two patch clamp pipettes used to record synaptic transmission are visible, one on a control neuron (bottom), the other on a transfected GFPpositive neuron (top). (b) Example of a typical recording. In response to stimulation of the NCS-1-transfected neuron (double arrows, top trace), EPSCs that showed PPF were recorded in the postsynaptic neuron (bottom trace). Conversely, when the non-transfected neuron was stimulated (double arrows, bottom trace), PPD was observed in the EPSCs 
recorded in the transfected neuron (top trace). Note the autaptic responses in the nontransfected neuron, showing depression (*). (c,d) Amplitudes of the first (closed symbols) and second (open symbols) EPSC over a 10-min recording period from an individual pair of neurons that showed reciprocal synaptic connections, stimulated every $30 \mathrm{~s}$ with a pair of pulses separated by $50 \mathrm{~ms}$. (c) Responses to stimulation of the control neuron (०); (d) responses to stimulation of the NCS-1-transfected neuron $(\boldsymbol{\nabla})$. (e) The same data as in $\mathbf{c}$ and d, but the amplitude of the second EPSC is now plotted against the amplitude of the first EPSC (ISI, $50 \mathrm{~ms}$ ). There was no correlation between the two for either the control (example shown, circles; slope $=0.06, \mathrm{r}^{2}=0.1$ ) or the NCS-1-transfected neurons (example shown, $\boldsymbol{\nabla}$; slope $=0.3, \mathrm{r}^{2}=0.16$ ). (f) Plot of average PPR versus average initial EPSC amplitudes in eight individual recordings for control ( $)$ and NCS-1-transfected synapses $(\boldsymbol{\nabla})$. 

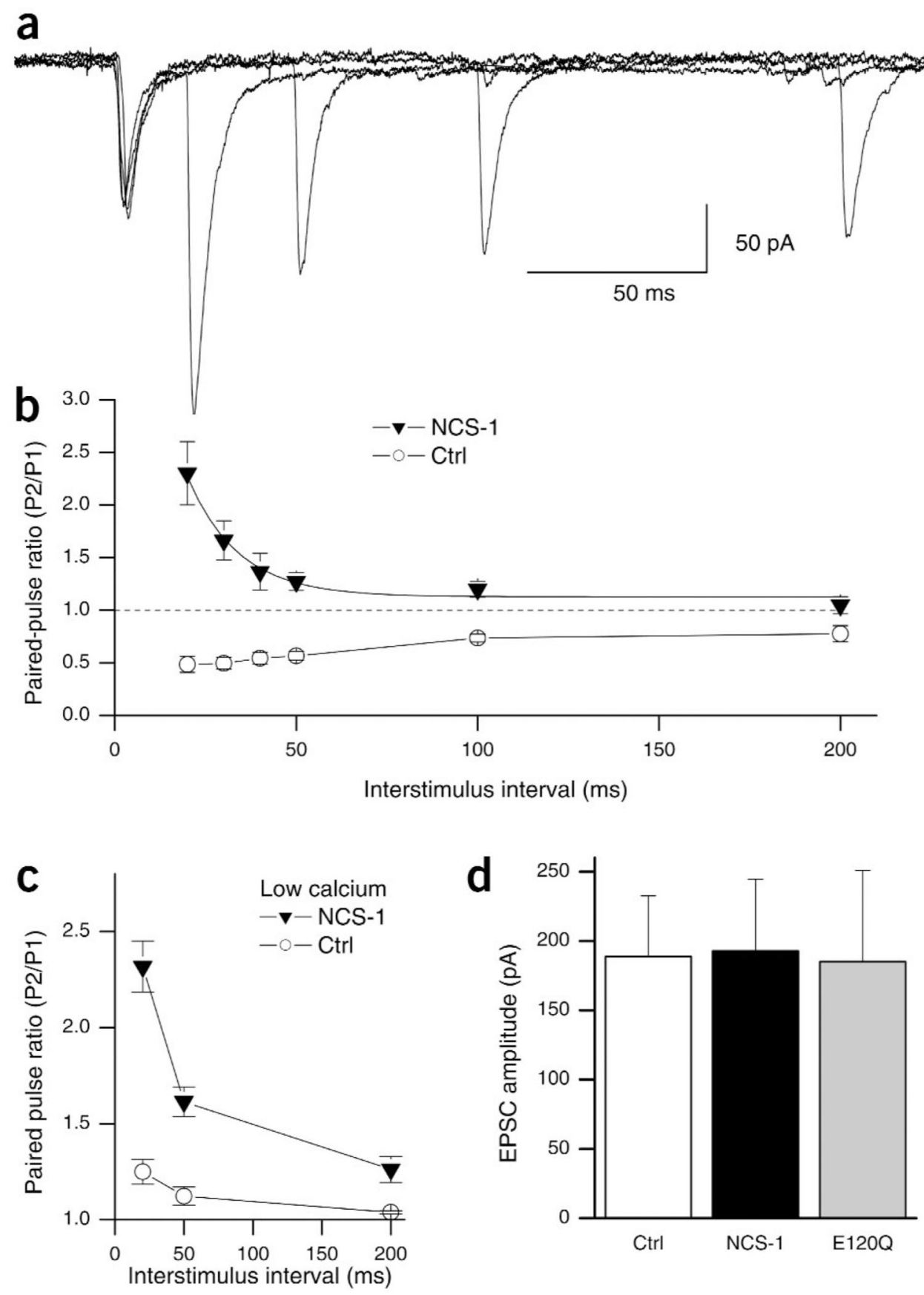

Figure 2.

NCS-1 expression confers facilitation, but does not affect basal neurotransmitter release. (a) Postsynaptic recordings from a pair of neurons in which an NCS-1 transfected neuron is stimulated at varying ISIs. Five traces at 20-, 30-, 50-, 100- and 200-ms ISI are superimposed. (b) Summary data showing average PPR at varying ISIs for control (O) and NCS-1 transfected synapses $(\boldsymbol{\nabla})$ in normal extracellular calcium $(2 \mathrm{mM})$. At NCS-1transfected synapses, facilitation is apparent at ISIs between 20 and $100 \mathrm{~ms}(P<0.01, n=6)$ and nonsignificant at $200 \mathrm{~ms}(P>0.2, n=6)$. The NCS-1 data was fit by a single exponential with a tau of $14 \pm 3 \mathrm{~ms}$ (solid line). (c) Summary data showing average PPR at varying ISIs for control $(\circ, n=8)$ and NCS-1-transfected synapses $(\boldsymbol{\nabla}, n=6)$ in low 
extracellular calcium (1 mM). Low calcium alleviated PPD in control synapses, but PPF at NCS-1-transfected synapses in low calcium was still enhanced relative to control $(P<0.05$ at all ISI). (d) Average amplitude (mean \pm s.e.m.) of the first EPSC in control, NCS- 1 and E120Q transfected neurons. Amplitudes are not significantly different from each other $(P>$ $0.8 ; n=10$ for control and NCS- $1 ; n=8$ for E120Q). 


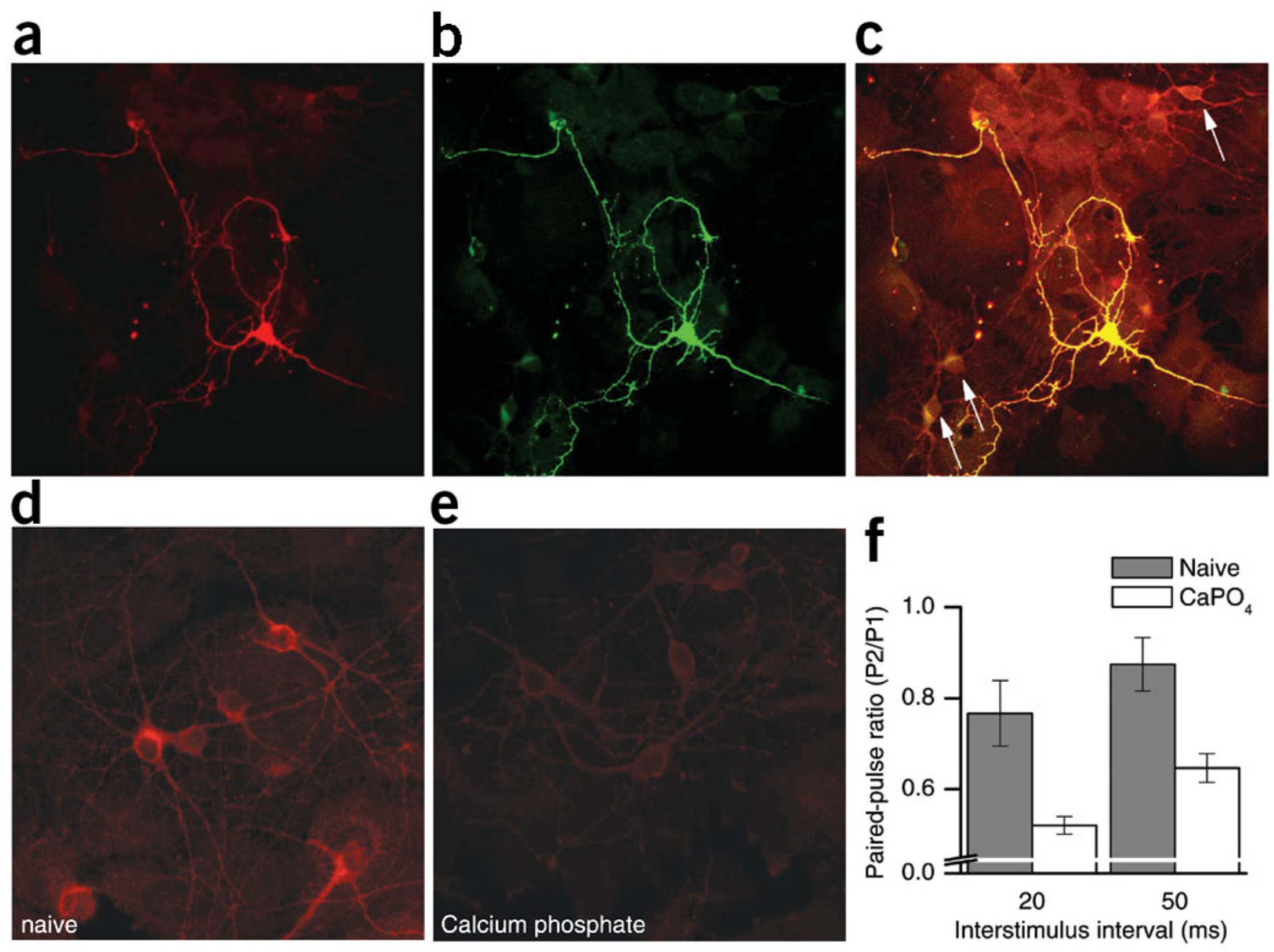

Figure 3.

NCS-1 expression correlates with short-term plasticity. We double-labeled pNCS1-IRESGFP transfected hippocampal neurons with antibodies against NCS-1 (a) and GFP (b). The image in $\mathbf{c}$ is a superimposition of images in panels $\mathbf{a}$ and $\mathbf{b}$, and has been overexposed to reveal the presence of non-transfected, GFP-negative neurons (arrows), which have low levels of NCS-1, comparable to adjacent glial cells. (d) Cultured hippocampal neurons labeled with antibodies against NCS-1 at 9 d.i.v. without calcium phosphate treatment. These 'naive' neurons clearly expressed NCS-1 at higher levels than the adjacent (barely visible) glial cells and higher than calcium phosphate-treated neurons shown in e stained with the same antibody. (f) PPD measured in pairs of neurons that had never been treated with calcium phosphate (naive, hatched bars, $\mathrm{PPR}_{20 \mathrm{~ms}}=0.77 \pm .07, \mathrm{PPR}_{50 \mathrm{~ms}}=0.87 \pm 0.06, n$ $=8)$ and in neurons mock-transfected with calcium phosphate but without DNA $\left(\mathrm{CaPO}_{4}\right.$, open bars, $\left.\mathrm{PPR}_{20 \mathrm{~ms}}=0.52 \pm 0.02, \mathrm{PPR}_{50 \mathrm{~ms}}=0.65 \pm 0.03, n=9\right)$. The differences in PPR are statistically significant $(P<0.01$ for both ISIs). 
a

Control

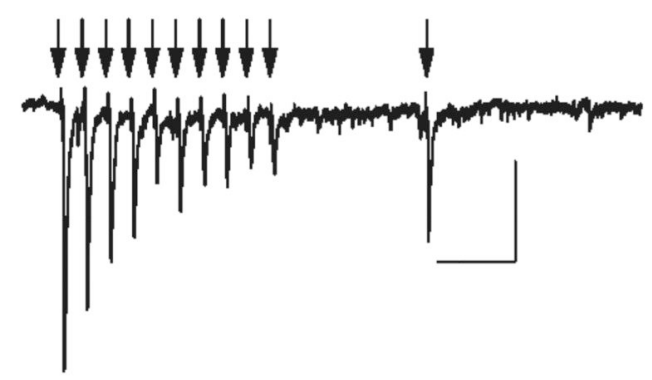

NCS-1
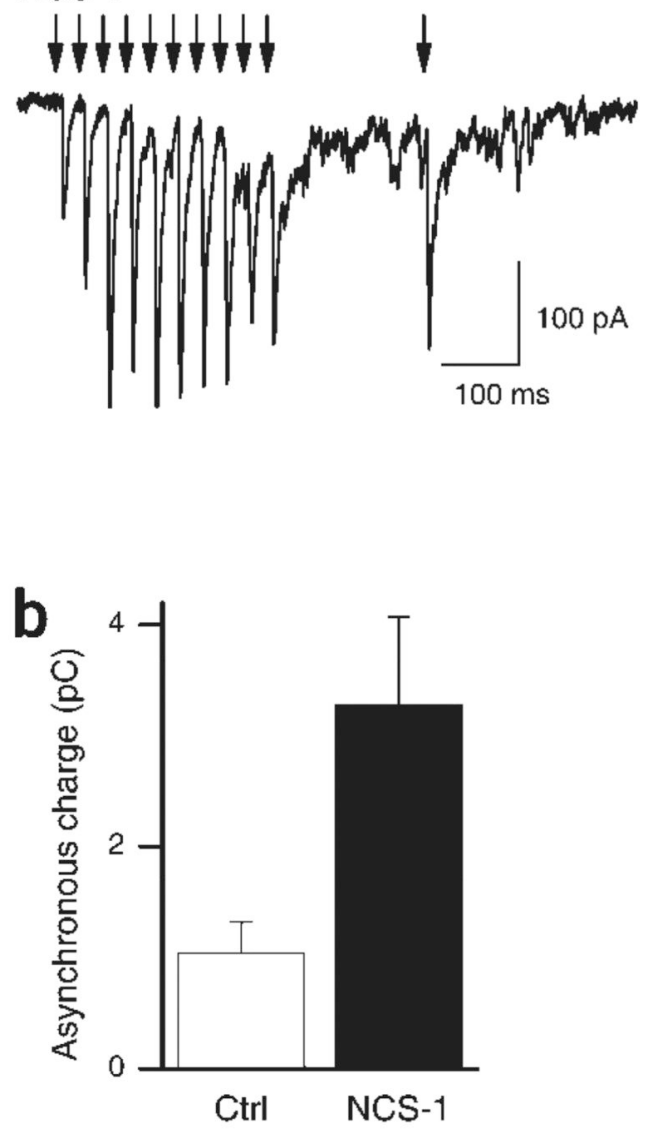

C

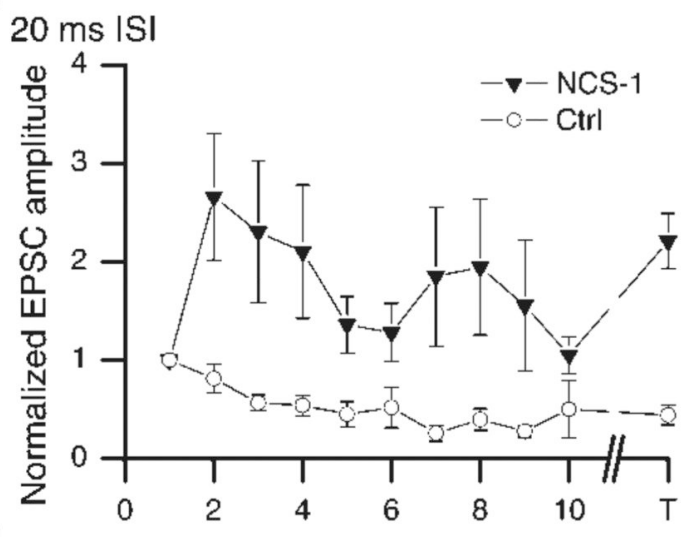

$\mathbf{d}_{30 \mathrm{~ms} \mid \mathrm{sI}}$

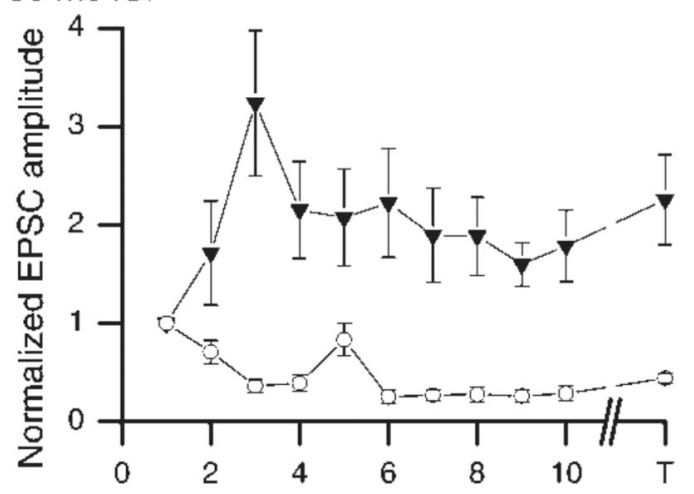

e

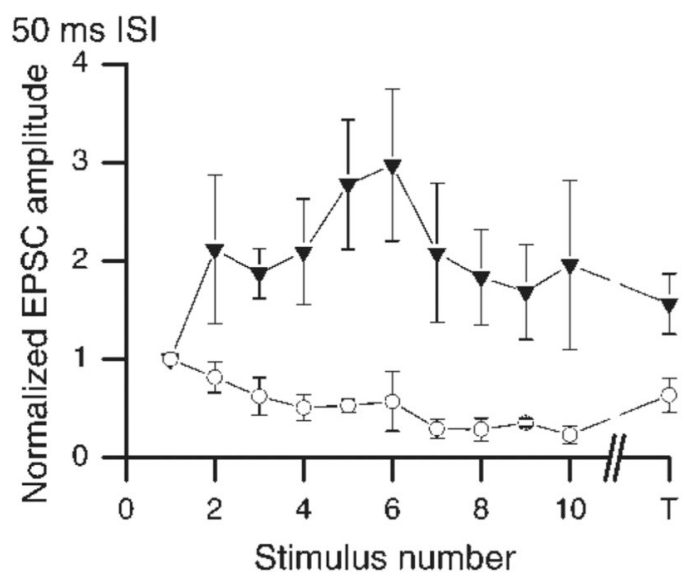

Figure 4.

Synaptic responses from NCS-1-transfected synapses remain facilitated during highfrequency stimulus trains. (a) Representative traces of EPSCs in response to a train of stimuli given at $33 \mathrm{~Hz}$ and a test stimulus given $200 \mathrm{~ms}$ after the train. Traces were taken from a bidirectional synapse in which EPSCs were elicited from both a transfected and nontransfected cell during the same recording. Top trace shows the depression when the control cell was stimulated, with only partial recovery at the time of the test pulse. Bottom trace shows EPSCs during a train at a transfected synapse, where the response was facilitated throughout the train and stayed potentiated at the time of the test pulse. Arrowheads indicate presynaptic stimulation. (b) Asynchronous release after the train was significantly enhanced. 
The current was integrated from $20 \mathrm{~ms}$ after the last stimulus for $180 \mathrm{~ms}$ (i.e., until the test stimulus). Control, $1.04 \pm 0.28 \mathrm{pC}, n=7$; NCS-1, $3.28 \pm 0.79, n=6 ; P<0.05$. (c-e)

Summary data of amplitudes (normalized to the first EPSC) for each stimulus (1-10) and the test stimulus (T) during trains at $50 \mathrm{~Hz}(\mathbf{c}), 33 \mathrm{~Hz}(\mathbf{d})$ and $20 \mathrm{~Hz}(\mathbf{e} ; n=6-8)$. 


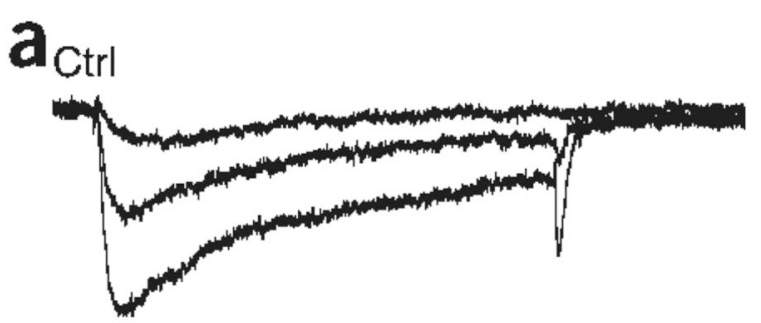

NCS-1

b

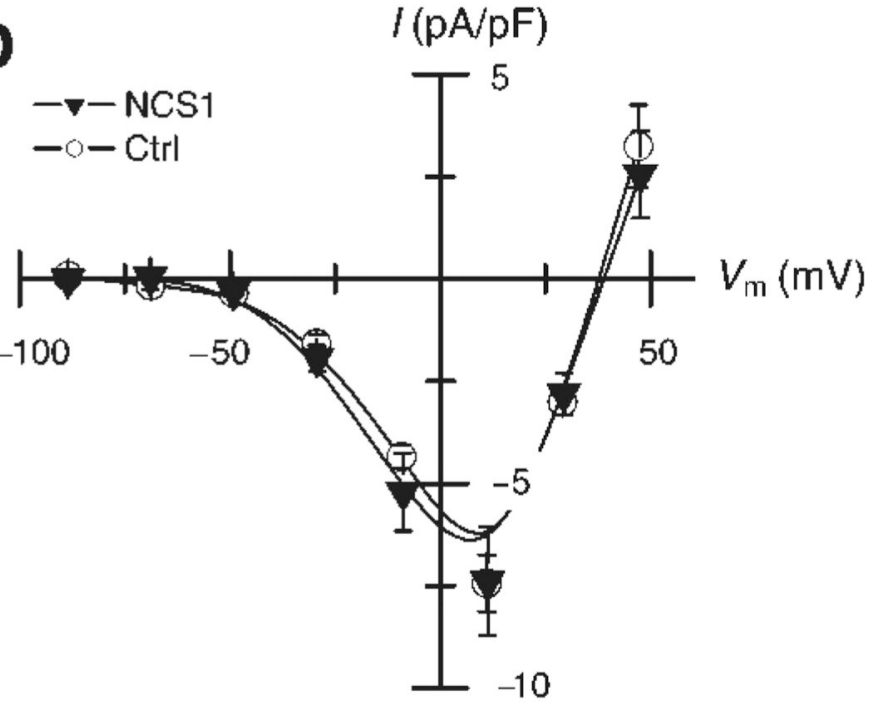

C

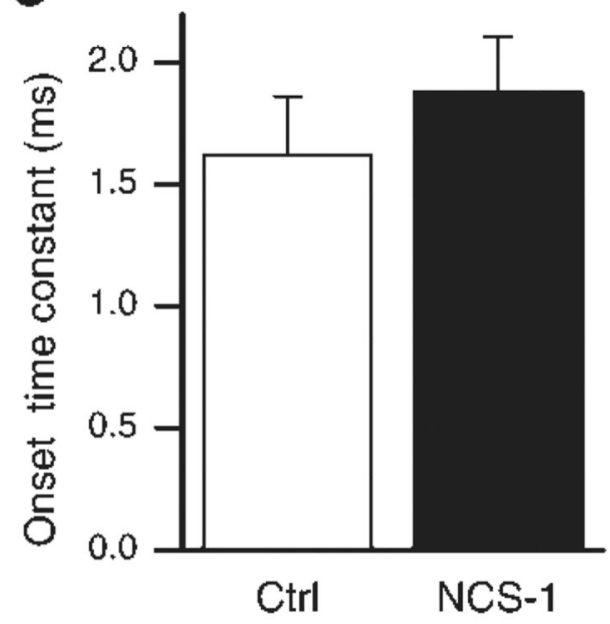

d

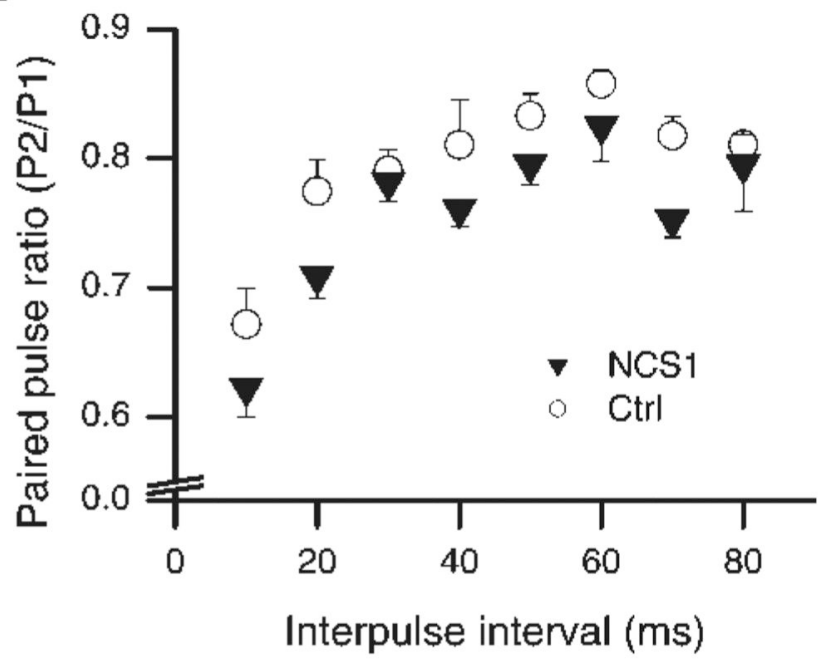

Figure 5.

Neither calcium current amplitudes nor kinetics are altered in NCS-1-transfected neurons. (a) Sample traces of calcium currents elicited by 100-ms depolarizations to $-30,-10$ and $+10 \mathrm{mV}$ in both control (top, averages of 3) and NCS-1-transfected (bottom, averages of 4) neurons. All traces are leak-subtracted. Experiments were done in the presence of nimodipine $(3 \mu \mathrm{M})$ to block L-type calcium channels (see Methods). (b) Current-voltage (I$V$ ) relationships of $I_{\mathrm{Ca}}$ measured at the peak of the response (a) and normalized to the membrane capacitance. Superimposed $I-V$ curves for control (O) and NCS-1 transfected neurons $(\boldsymbol{\nabla})$ show almost complete overlap. The interpolated reversal potential is unchanged (control, $+38 \mathrm{mV}$; NCS-1, $+39 \mathrm{mV} ; n=7$ for both groups). (c) The onset time constant of $I_{\mathrm{Ca}}$ at $+10 \mathrm{mV}$ for control (black bar) and transfected (open bar) cells were 1.62 $\pm 0.24 \mathrm{~ms}$ and $1.88 \pm 0.68 \mathrm{~ms}$, respectively, and not significantly different $(P>0.4 ; n=6$ for control, $n=9$ for transfected). (d) Summary data showing average PPR for $I_{\mathrm{Ca}}$ at $+10 \mathrm{mV}$ at varying ISIs. PPD of $I_{\mathrm{Ca}}$ is observed at all intervals tested $(10-80 \mathrm{~ms})$ for both control and 
NCS-1-overexpressing groups, and is not significantly different $(P>0.5, n=7$ for both groups). 

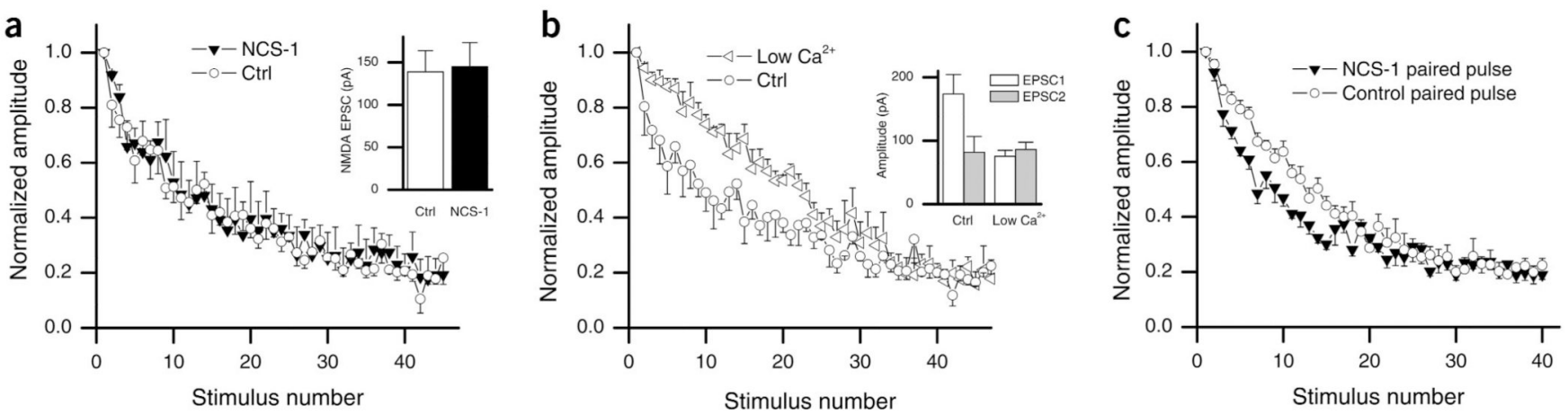

Figure 6.

Rate of MK-801 blockade is not altered at NCS-1-transfected synapses. (a) The progressive block by MK-801 of the NMDA receptor EPSC is shown as a plot of amplitude versus time in both transfected and non-transfected neurons stimulated every $15 \mathrm{~s}$. Amplitudes shown are normalized to the first response after bath application of MK801. Averaged data from control ( $($ ) and NCS-1-transfected neurons $(\boldsymbol{\nabla})$ showed no difference in the rate of block $(n$ $=7$ for both groups; $P=0.62$ for pulses $1-20 ; F_{1,19}=0.241$, two-way ANOVA). Inset, average amplitudes of NMDA-mediated EPSCs show no significant difference in amplitude ( $P>0.7, n=5$ transfected; $n=4$ control). (b) Rate of MK-801 block is significantly slowed by reduction of external calcium from $2 \mathrm{mM}(\circ)$ to $1 \mathrm{mM}(\triangleleft ; n=7$ for both groups; $P<$ 0.001 for pulses $1-20 ; F_{1,19}=309.1$, two-way ANOVA). Inset, average first and second EPSC with a 50-ms ISI in control calcium ( $2 \mathrm{mM}$, first response: $174 \pm 30 \mathrm{pA}$, second response: $82 \pm 25, \mathrm{n}=10)$ and in low calcium $(1 \mathrm{mM}$, first response: $75 \pm 9 \mathrm{pA}$, second response: $86 \pm 11, n=8$ ). (c) Rate of MK-801 block was tested using pairs of stimuli in both the control and transfected conditions. Cells were given a pair of stimuli (with a 50-ms ISI) every $30 \mathrm{~s}$. Amplitudes shown are of the first EPSC of each stimulation pair normalized to the first amplitude after bath application of MK-801. The rate of block in the NCS-1transfected neurons (filled squares) was significantly faster than in control $(\circ)$, indicating an increased release probability on the second stimulus ( $n=5$ for both groups; $P<0.001$ for pulses $1-20 ; F_{1,19}=82.3$, two-way ANOVA). 

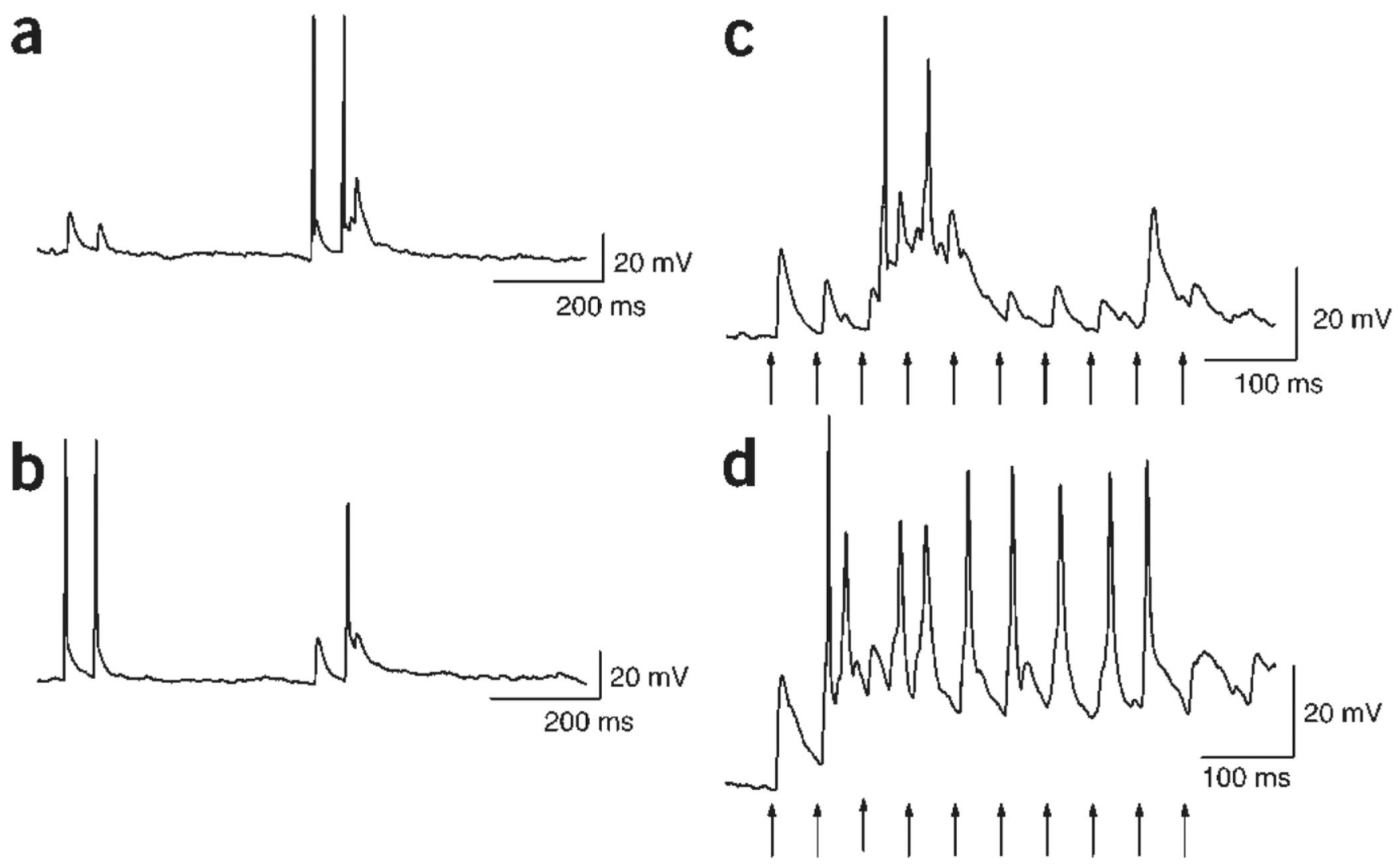

Figure 7.

Current clamp experiments show that NCS-1 confers more reliable postsynaptic firing. (a,b) Example traces of a typical current clamp recording from reciprocally connected pair of neurons. Stimulation of the control neuron with a pair of stimuli (50-ms ISI) elicits EPSPs in the transfected neuron (a) that show PPD. (b) Conversely, when the NCS-1-transfected neuron was stimulated, PPF was observed in the EPSPs recorded in the non-transfected neuron, sufficient to elicit an action potential. (c,d) Representative traces of postsynaptic responses during a train of stimuli (10 pulses at $20 \mathrm{~Hz}$, arrows). Stimulation of the control neuron elicits EPSPs in the postsynaptic cell (c) that depress during successive stimuli. In this example, the third EPSP is followed by action potentials that probably are triggered by other neurons in the network. However, even with the help of this additional excitation, none of the experimentally triggered responses were able to depolarize the neuron above threshold. Stimulation of the NCS-1-transfected neuron facilitated EPSPs, which elicited a postsynaptic action potential for almost every stimulus (d). 